\title{
Aqueous electrophoretic deposition as a method for producing an investment casting shell mould ceramic face-coat. Part 1: formation of a carbon-filled investment casting wax electrode material
}

\author{
P. J. Roach • C. B. Ponton
}

Received: 6 May 2013/Accepted: 26 June 2013/Published online: 20 July 2013

(c) The Author(s) 2013. This article is published with open access at Springerlink.com

\begin{abstract}
Aqueous electrophoretic deposition (EPD), using a zircon, i.e., zirconium silicate $\left(\mathrm{ZrSiO}_{4}\right)$ suspension, has been investigated as an alternative to the conventional slurry dip-coating process for producing the face-coat of investment casting ceramic shell moulds. This is because EPD has the potential to (a) increase dimensional tolerances in the resultant casting, and (b) form a uniform facecoat on the entire mould-surface of complex shell moulds, including the small and/or complex cavities that are a problem for conventional dip-coating. Part 1 of this work addresses the formation of carbon-filled investment casting wax composite electrode materials. A carbon black powder and a micronised graphite powder were used as the alternative fillers in a water-emulsified pattern wax, an unfilled pattern wax and an unfilled runner wax. The runner wax composites exhibited consistently higher resistivities for both filler types and across the range of filler concentrations. Electrical resistivities of 1200 and $240 \Omega \mathrm{cm}$ were attained for the $16 \mathrm{vol} \%$ micronised graphite-filled and carbon black-filled straight pattern wax composites, respectively. The higher conductivity values associated with the use of carbon black filler are attributed to its highsurface area and hierarchical agglomerated structure.
\end{abstract}

\section{P. J. Roach}

European Mill Products (Kitts Green), Alcoa Europe, Kitts Green Road, Kitts Green, Birmingham B33 9QR, UK

C. B. Ponton $(\bowtie)$

School of Metallurgy and Materials, College of Engineering and Physical Sciences, University of Birmingham, Edgbaston, Birmingham B15 2TT, UK

e-mail: c.b.ponton@bham.ac.uk

URL: http://www.birmingham.ac.uk/schools/metallurgymaterials/people/profile. aspx ?ReferenceId $=6742 \&$ Name $=\mathrm{dr}-$ clive-ponton
Rheologically, the micronised graphite-filled runner wax and water-emulsified pattern wax composites followed the Krieger-Dougherty model, which is helpful given that investment casting waxes are invariably injection moulded. In summary, these results confirm, in conjunction with Part 2, the feasibility of developing EPD as a promising technique for forming the investment casting shell mould ceramic face-coat.

\section{Introduction}

The production of complex investment cast metal components requires equally complex and accurately dimensioned ceramic shell moulds. For example, highperformance aerospace turbine blades not only have a complex external geometry, but also have small intricate internal air-cooling channels. Fabricating complex internal cavity geometry moulds invariably requires the use of removable ceramic cores. The mould is formed by successive slurry dip-coating (investment) and stuccoing stages. These stages deposit the appropriate ceramic coatings on the pre-patterned substrate, starting with the primary or face-coat. The substrate material must be then easily removable by melting, or dissolution, to leave behind the hollow refractory ceramic shell mould. Thus, specialised waxes are currently used as the substrate material.

However, the need for internal cores increases the number of stages in the investment casting process, as well as introducing potential problems. The problems include reactivity between the ceramic core and the metal; a decrease in the internal cast surface quality; internal structures with poor dimensional tolerances; and ceramic core breakage and/or displacement during casting. Furthermore, as the 
internal cavity geometries within an investment casting ceramic shell mould become more complex and/or smaller, the ability to directly and uniformly dip-coat the cavity surfaces decreases.

Hence, the feasibility of employing an environmentfriendly, i.e., aqueous, electrophoretic deposition (EPD) process for producing the face-coat has been investigated. The reason being that EPD has the ability to deposit a uniform ceramic coating onto an appropriate substrate material from an aqueous suspension of ceramic particles. EPD also has the potential to (a) increase dimensional tolerances in the resultant casting and (b) form a uniform face-coat on the entire mould-surface of complex shell moulds, including the small and/or complex cavities that are a problem for conventional dip-coating. This offers the possibility of eliminating the need for some ceramic cores and the attendant problems their usage could bring.

In order for an aqueous EPD process to replace, or at least, be an alternative to the conventional primary dipcoating step of investment casting, it is essential that the substrate pattern material developed can replicate what investment casting waxes achieve within the current industrial investment casting process, and yet be electroconductive. This is because electrophoretic deposition takes place due to the presence of an electric field within the suspension medium, which attracts charged particles in suspension towards an electrode of opposite charge. These particles are then deposited onto this electrode to form a coating.

Devising such an EPD process required (a) the formation of investment casting wax composite electrode materials (addressed in Part 1), and (b) the development of a zircon, i.e., zirconium silicate $\left(\mathrm{ZrSiO}_{4}\right)$ powder aqueous suspension suitable for use in the EPD process (addressed in a future Part 2 paper), employing the formed electroconductive wax materials as the deposition electrode.

Approaches to fabricating an electroconductive meltout or dissolve-out investment casting pattern EPD substrate material system

There are three main approaches to the fabrication of an electroconductive melt-out or dissolve-out investment casting pattern EPD substrate material system:

Approach 1: Employ, if possible, an aqueous-based (for environmental reasons) electroconductive coating that is easy to apply to the surface of the electrically non-conductive standard investment casting pattern material prior to the EPD stage being carried out. The electroconductive coating would then be subsequently removed together with the pattern material from the shell mould. Using this method, the standard investment casting pattern material could be used, albeit with modified pattern dimensions to make allowance for the electroconductive coating thickness. This would enable the current industrial investment casting stages on either side of the ceramic coating steps to be carried out with only minor alterations. There are examples in the literature of the deposition of electroconductive coatings [1-3]. However, achieving electroconductive coating thickness uniformity on complex geometries remains an issue.

Approach 2: Incorporate a reusable highly electroconductive core electrode material within the electrically non-conductive standard investment casting pattern material, for the entire pattern surface to act as the EPD substrate. However, the core electrode material must be capable of generating an electric field high enough to overcome the masking effect of the pattern material that surrounds it, enabling successful EPD. Achieving a uniform field strength everywhere on the complex geometry substrate surface will most likely require the manufacture of relatively complex-geometry core electrode designs derived from electric field versus electrode geometry modelling research.

Approach 3: Modify the electrically non-conductive standard investment casting pattern material by incorporating an electroconductive filler phase, which does not prevent the matrix material from being melted out or dissolved out as normal, to make it electrically conductive. As well as using waxes, resins or polymers containing electroconductive fillers, electrically conducting polymers or polymer blends could also be used to form an EPD substrate. Furthermore, it is not difficult to combine these latter two approaches, since the electroconductive pattern material could be formed with a reusable more highly electroconductive simple-geometry core incorporated.

A comparison of these alternative approaches to the fabrication of an electroconductive investment casting pattern EPD substrate material in Table 1 indicates that Approach 3 would be the simplest approach for the investment casting industry to employ. For this reason, the 
Table 1 Comparison of approaches to the fabrication of an electroconductive melt-out or dissolve-out investment casting pattern EPD substrate material system

\begin{tabular}{|c|c|c|}
\hline & $\begin{array}{l}\text { Modifications required to } \\
\text { standard wax pattern materials } \\
\text { by wax manufacturers prior to delivery } \\
\text { to investment casting companies }\end{array}$ & $\begin{array}{l}\text { Additional stages required as part of the } \\
\text { investment casting process carried out by } \\
\text { investment casting companies, in addition } \\
\text { to the EPD process itself }\end{array}$ \\
\hline Approach 1 & None & $\begin{array}{l}\text { Sourcing and application of an aqueous } \\
\text { solution-based (for environmental reasons) } \\
\text { electroconductive coating onto each } \\
\text { wax pattern and the provision of an electrical } \\
\text { connection between the coating and the } \\
\text { EPD circuit set-up }\end{array}$ \\
\hline Approach 2 & None & $\begin{array}{l}\text { Design, manufacture and incorporation } \\
\text { of a probably relatively complex-geometry, } \\
\text { highly conductive core electrode into } \\
\text { each wax pattern }\end{array}$ \\
\hline Approach 3 & $\begin{array}{l}\text { Incorporate electroconductive } \\
\text { filler in the wax materials }\end{array}$ & $\begin{array}{l}\text { Incorporation of a simple-geometry } \\
\text { electrical connection tab (for connecting } \\
\text { to the EPD-circuit setup) within each } \\
\text { electroconductive wax pattern during } \\
\text { its fabrication }\end{array}$ \\
\hline
\end{tabular}

present work was focussed on developing an electroconductive filler phase-investment casting wax matrix composite material.

Nature of the electroconductive filler phase in the investment casting wax matrix composite material

The use of a metallic electroconductive filler phase is precluded because the possible retention of certain metallic species within the investment shell face-coat during the EPD process could have a very detrimental effect on the resulting cast metal properties. Thus, in the present work, a carbon-based filler phase was utilised. The choice of carbon filler for the present work was restricted to acceptably low cost, commercially available carbon materials, namely, graphite and carbon black powders. The alternative materials, such as carbon nanofibres, or carbon nanotubes, are too expensive at present to be considered commercially by the investment casting industry.

In contrast to previous studies on carbon-polymer composites that employed a single polymer, or a paraffin wax matrix [4-7], the necessary use of a modern investment casting wax as the matrix adds a further complicating factor to the carbon-wax composite behaviour analysis. This is because modern investment casting waxes are very complex low-melting point (typically less than $85^{\circ} \mathrm{C}$ ) designed-forpurpose materials that contain numerous components [8].

Another factor that needs to be considered is the carbonwax composite electroconductivity, as melt-out matrices containing conductive filler particles need to be conductive enough for EPD coating to form on the substrate's surface. Thus, the phenomenon of percolation, i.e., the connectivity of one substance or phase within the matrix of another, needs to be addressed. Whilst percolation theory is often used to model fluid flow through porous media, the theory can be applied in the present work to the flow of electric current, as conducting particles form infinite bridges in an insulating medium. The percolation threshold $\left(P_{\mathrm{c}}\right)$ here corresponds to the point where the conducting particles are at a high enough concentration to form a connecting lattice, i.e., there is long-range connectivity, and this is shown by a significant decrease in the sample's resistance with increasing conductor phase content [9]. Conductor-insulator composite materials are often used for modelling percolation behaviour, where the model lattice can be viewed [10] as being constructed in terms of sites (which are bond intersections) and bonds (which are pairwise connections between sites).

This construct gives rise to a critical site probability (percolation threshold), $P_{\mathrm{cs}}$, and a critical bond probability (percolation threshold), $P_{\mathrm{cb}}$. The percolation threshold for a system depends on its dimensionality, lattice type and the percolation type (i.e., site or bond percolation). While the values of $P_{\mathrm{cs}}$ and $P_{\mathrm{cb}}$ differ for each three-dimensional (3D) lattice type, e.g., $P_{\mathrm{cb}}$ is 0.119 for face-centred cubic (fcc), 0.179 for body-centred cubic (bcc), and 0.247 for simple cubic (sc) lattices, the key point to note is that the critical (percolation) volume fraction of the high-conductivity phase for $3 \mathrm{D}$ random packing is almost constant, changing relatively little with 3D lattice type. The average based on the values for fcc, bcc, sc, diamond, and random close packed lattices is $0.16 \pm 0.02$, assuming purely spherical conducting and insulating particles equal in dimension, with no size distribution [10]. Nevertheless, this is a useful result because practical composite materials are usually 3D, given that the smallest composite sample dimension is very much larger than the characteristic dimensions of the largest microstructural features, e.g., particles, grains, etc., in the microstructure. 
Scarisbrick [11] usefully modelled the probability of conduction occurrence within unit cube models, considering three possible distributions of the conductive particles: separate, adjacent and touching, and compared the model predictions against the behaviour of carbon black-polymer composites and particle mixtures. Kusy [12] showed that dispersions of one powder within another have properties influenced by both the particle size ratio and the volume fraction. Thus, varying the particle size ratio $\lambda=d_{\mathrm{i}} / d_{\mathrm{c}}$ (where $d_{\mathrm{i}}$ is the diameter of the insulating particle and $d_{\mathrm{c}}$ is the diameter of the conducting particle) makes the mixture either random or segregated in nature, as shown in Fig. 1 . The critical volume percent, $V_{\mathrm{c}}$ is the volume fraction required for the dispersed powder phase to become continuous, and according to this model, plotting the critical volume percent against the particle size ratio gives an exponential curve. This means that decreasing $d_{\mathrm{c}}$ within a system with a fixed insulating particle diameter results in a smaller critical volume percent. $V_{\mathrm{c}}$ and $P_{\mathrm{c}}$ are only equivalent when the dispersed powder phase is randomly distributed throughout the matrix.

Finally, other factors to be aware of and consider are: (a) mass segregation of the carbon filler phase within the wax due to density differences; (b) carbon-filled waxes modifying the wax injection rheology; and (d) ash content of carbon-filled waxes hindering the de-waxing process, with potential surface defect generation in the cast metal.

\section{Experimental details}

Characterisation of as-received electroconductive powders

A carbon black and a micronised graphite powder were individually employed as an electroconductive filler material. The carbon black powder was Vulcan XC605 (Cabot Co.), which is a 'chemically clean' form of carbon black powder produced specifically for electrostatic discharge (ESD) applications requiring enhanced electrical conductivity. Carbon black is chemically and physically distinct from soot and black carbon [13], and as a powder, it comprises a hierarchy of increasing-size structures covering the size range $\sim 15$ to $\sim 100 \mu \mathrm{m}[7,13]$. The micronised graphite powder was a high purity $(99.5 \%)$ natural crystalline graphite powder, described by the manufacturer (Graphite Trading Co.) as having particles with diameters of less than $10 \mu \mathrm{m}$.
Fig. 1 Bi-powder dispersions represented in two-dimensions, within an elementary environment, D. The dark circles represent spherical conducting particles, whilst the light circles represent spherical insulating particles. $\mathbf{a}$ is a system where $d_{\mathrm{i}} / d_{\mathrm{c}}=1$ and $V<V_{\mathrm{c}}$. $\mathbf{b}$ is a system where $d_{\mathrm{i}} / d_{\mathrm{c}}=1$ and $V>V_{\mathrm{c}}$. $\mathbf{c}$ is a system where $d_{\mathrm{i}} / d_{\mathrm{c}}=5$ and $V<V_{\text {c }}$. d is a system where $d_{\mathrm{i}} / d_{\mathrm{c}}=5$ and $V>V_{\mathrm{c}}$ (a)

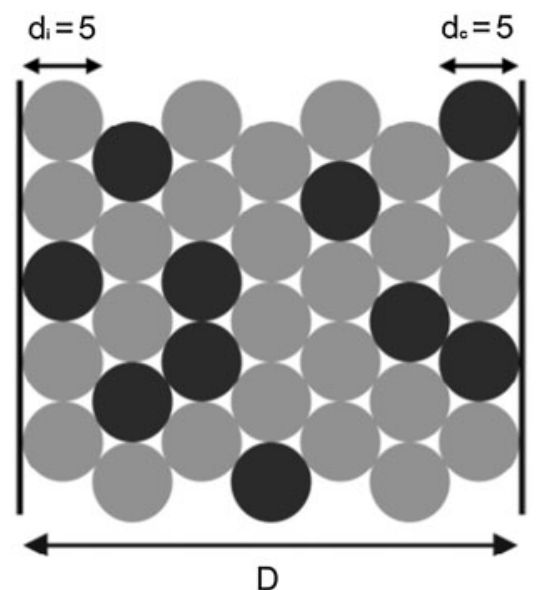

(c)

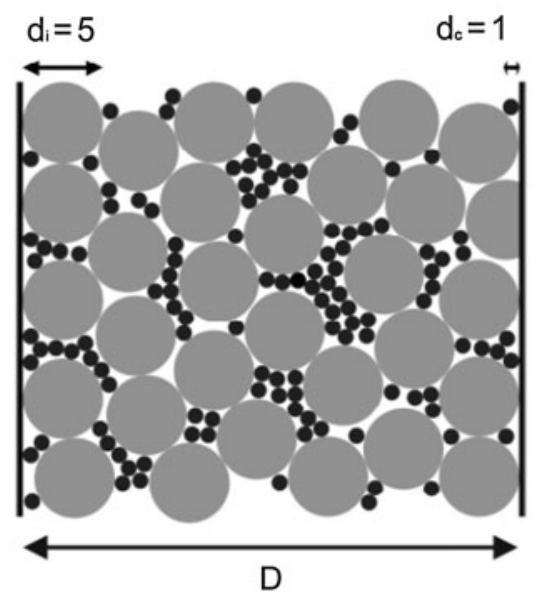

(b)

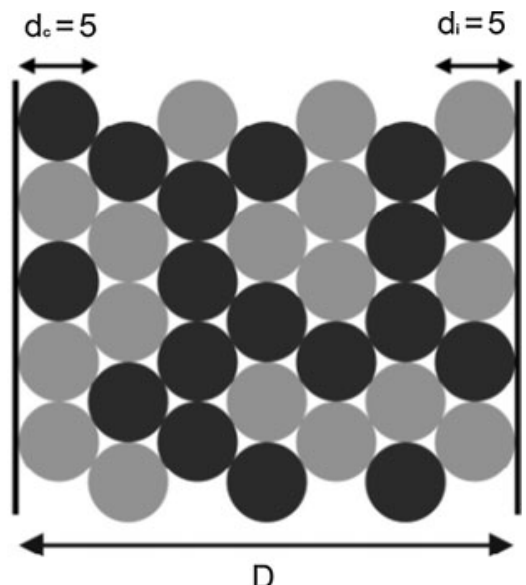

(d)

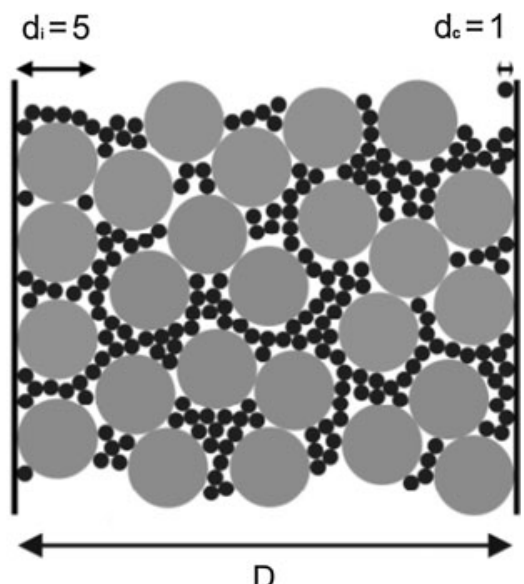


The powder particle size distribution, morphology, and the level of agglomeration all have a strong influence on how well the particles become dispersed within the wax matrix. These particle characteristics, in conjunction with the particle dispersion, determine the flow behaviour of the carbon-wax composite and its electrical conductivity. Hence, the powder surface area, particle/agglomerate size distribution and particle morphology were investigated. Powder surface area analysis was done via the multipoint BET method using a Micromeritics ASAP 2010 Accelerated Surface Area and Porosimetry System (determining nitrogen adsorption and desorption isotherms at liquid nitrogen temperature and relative pressures $\left(P / P_{\mathrm{o}}\right)$ ranging from 0.05 to 1.0 ). Secondary electron imaging (SEI) mode scanning electron microscopy (SEM) was used to observe the particle/agglomerate morphology.

The carbon powder particle/agglomerate size distributions were measured using laser diffraction particle size analysis (Beckman Coulter LS320 with a lower size measuring limit of $40 \mathrm{~nm}$ ), following the Beckman Coulter recommended instrument preparation and calibration procedures.

Owing to the non-dispersal of both carbon black and graphite powders in water, reagent-grade ethanol was used as the suspending medium. Furthermore, due to the noted tendency of the carbon powders to agglomerate in suspension due to intermolecular interactions, half of the suspension samples underwent ultrasonication, using a Jencons VCX 600 ultrasonic processor with a 13-mm solid probe tip. For each prepared suspension, a $0.5 \mathrm{~cm}^{3}$ volume was taken from the suspension using a pipette, and this was added to $4.5 \mathrm{~cm}^{3}$ of ethanol (reagent-grade $99 \%$ ) in a specimen container. This dilution reduced the particle concentration to a level suitable for analysis. The suspension fluid was described as being ethanolic for the purposes of software calculations, and Mie theory was used to analyse the particle sizing data, as the alternative Fraunhofer approximations are not suited for use with particle size distributions containing non-spherical material less than $2 \mu \mathrm{m}$ in size, which is the case here. For each diluted suspension tested, three samples were analysed, and an average of these was taken to form a particle size distribution curve if no anomalous behaviour was observed.

\section{Wax matrix materials}

Three investment casting pattern wax grades from Blayson Olefines Ltd., and studied as electroconductive carbon-wax composite matrices:

A7-11 unfilled (straight) pattern wax

A7-7808 unfilled (straight) runner wax

A7-TC2/E water-emulsified wax (water content of $8-10$ vol\%)
The nominal properties of these grades according to the manufacturer, Blayson Olefines Ltd., are tabulated in Table 2.

The A7-11 wax was chosen initially as it is a commonly used wax grade with properties that ideally suit it for use without any need to change current wax injection moulding parameters. However, the addition of electroconductive filler markedly increased the working temperature range and viscosity of the A7-11 wax matrix composite materials; thus, it was effectively only used in the experiments described in the "Electrical resistivity measurements" section for the reasons explained in the "Rheology measurements" section. Thus, the A7-7808 wax was chosen so that the increase in working temperature range and viscosity when filled with electroconductive filler could be offset against its lower unfilled working temperature range and viscosity. The A7-TC2/E wax grade was chosen in response to the mixing difficulties associated with the presence of carbon black filler particle agglomerates; it being hoped that the presence of water as a filler would aid the dispersion of both graphite and carbon black within the matrix, to produce a homogenous electroconductive wax composite with more uniform characteristics.

Formation of carbon-filled wax matrix composite material

The micronised graphite and carbon black powders were individually employed as an electroconductive filler material to form electroconductive networks within each of the three wax matrices, giving six carbon filled-wax composite materials for comparison. No material characterisation, nor any further processing, was carried out on the assupplied wax materials prior to the carbon filled-wax composite materials' sample formation.

The electroconductive wax samples were cast into three different geometries; flat circular discs, flat rectangular plates, and hollow hemispheres. The discs were made for use as electrical resistivity measurement samples, while the flat rectangular plates and hollow hemispheres were made for the zircon EPD experiments. The electroconductive wax electrical resistivity measurement samples were formed in flat-bottomed silicone rubber mould vessels placed on a magnetic stirrer thermistor controlled hotplate, at a temperature of $80-85{ }^{\circ} \mathrm{C}$. The wax pellets were allowed to fully melt prior to carbon powder addition. Magnetic and manual mixing methods were used in conjunction to aid filler distribution, and mixing was continuous until the carbon-wax composites samples were cast into pre-heated silicone rubber moulds. For analysis purposes, the electroconductive waxes were formed, when molten, into flat disc geometries with a $65-\mathrm{mm}$ diameter and $3.0-\mathrm{mm}( \pm 0.5 \mathrm{~mm})$ thickness. The wax-containing 
Table 2 Investment casting wax property data from Blayson Olefines Ltd.

\begin{tabular}{|c|c|c|c|c|c|c|c|}
\hline $\begin{array}{l}\text { Wax } \\
\text { grade }\end{array}$ & Filler & $\begin{array}{l}\text { Penetration } \\
\left(10^{-1} \mathrm{~mm}\right. \\
@ 100 \mathrm{~g})\end{array}$ & $\begin{array}{l}\text { Viscosity over } \\
\text { injection range } \\
(\mathrm{Pa} \mathrm{s})\end{array}$ & $\begin{array}{l}\text { Liquid } \\
\text { injection } \\
\text { range } \\
\left({ }^{\circ} \mathrm{C}\right)\end{array}$ & $\begin{array}{l}\text { Congealing } \\
\text { point }\left({ }^{\circ} \mathrm{C}\right)\end{array}$ & $\begin{array}{l}\text { Drop } \\
\text { melt } \\
\text { point } \\
\left({ }^{\circ} \mathrm{C}\right)\end{array}$ & Fluidity \\
\hline A7-11 & $\begin{array}{l}\text { None } \\
\text { present }\end{array}$ & 1-4 (hard) & $0.8-1.3$ (low) & $74-78$ & $69-73$ & $73-76$ & Medium \\
\hline A7-7808 & $\begin{array}{l}\text { None } \\
\text { present }\end{array}$ & 9-14 (medium) & $0.8-1.4$ (low) & $72-77$ & $62-67$ & $68-75$ & $\begin{array}{c}\text { Medium } \\
\text { Low }\end{array}$ \\
\hline A7-TC2/E & $\begin{array}{l}\text { Water } \\
6-8 \%\end{array}$ & 8-11 (medium) & 2.9-3.2 (high) & $67-71$ & $63-67$ & $72-75$ & $\begin{array}{c}\text { Medium } \\
\text { Low }\end{array}$ \\
\hline
\end{tabular}

silicone rubber disc mould was flipped over manually as it cooled to inhibit filler particle settling within the wax under gravity. A series of samples were formed with electroconductive filler contents ranging from 0 to 20 volume $\%$ (vol\%) since preliminary experiments indicated that a homogenous composite could not be formed when the graphite powder content was greater than $20 \mathrm{vol} \%$, and when the carbon black powder content was greater than $16 \mathrm{vol} \%$.

\section{Electroconductive wax composite analysis}

\section{Electrical resistivity measurements}

The sample material volume or bulk electrical conductivities were measured in terms of their volume or bulk electrical resistivity using a four-point probe technique; the lower the resistivity, the higher the conductivity since conductivity is the reciprocal of resistivity. For resistivity calculations, the samples would ideally be semi-infinite, i.e., the sample extends for all intents and purposes to infinity in all directions (and hence sample dimensions) below the plane upon which the four probes are to be located because then the true sample volume or bulk resistivity, $\rho(\Omega \mathrm{m})$ is given by [14] as

$\rho=2 \pi s\left(\frac{V}{I}\right)$

where $2 \pi s$ is the geometric factor for a semi-infinite volume of material, $s$ is the probe tip spacing (m), $V$ is the measured voltage $(\mathrm{V})$ and $I$ is the applied current (A). When this situation does not obtain, a geometric correction factor dependent on sample shape and dimensions, and the spatial arrangement of the electrical contacts (i.e., electrical probes) relative to each other and the sample has to be applied to Eq. 1. This is because when any sample boundaries other than the plane upon which the four probes are to be located are not an infinite distance from this plane and each other, they will restrict the possible paths of the electric current.

The Semiconductor Division of Haldor Topsoe has developed models for calculating four-point probe resistivities based upon thin and thick sample geometries [15]; for the sample geometries produced in the present work, their model and hence equation for thick samples, with its associated correction factors, was applicable. Thus, the sample resistivity values were calculated using

$\rho=G\left(\frac{V}{I}\right)$

where $\rho$ is the volume or bulk resistivity $(\Omega \mathrm{m}), V$ is the measured voltage (V), $I$ is the applied current (A), and $G$ is a geometric correction factor given by

$G=2 \pi s T_{1}\left(\frac{t}{s}\right)$

where $T_{1}(t / s)$ is an additional correction factor function required to account for the finite thickness of the sample that tends to 1 as $t$ approaches infinity, i.e., $T_{1}$ is a function of $t / s$, $t$ being the sample thickness (m) and $s$ being the probe tip spacing $(\mathrm{m})$. When $t \geq 5 \mathrm{~s}$, the geometric factor $2 \pi \mathrm{s}$ for a semiinfinite volume of material is correct to within $0.7 \%$ [15].

A resistance meter was set up with a linearly positioned tip spacing of $1 \mathrm{~mm}$. The probe set-up was placed in $10 \mathrm{set}$ positions on each sample. The probe was located so that the linearly positioned tips were always more than $20 \mathrm{~mm}$ from any lateral edge. The upper measurable limit for the apparatus was $100 \mathrm{k} \Omega$, which corresponds to a bulk resistivity of approximately $60 \mathrm{k} \Omega \mathrm{cm}$ for a 2.5 -mm thick planar sample. Thus, the resistivity of the unfilled waxes could not be experimentally verified because paraffin waxes generally possess volume resistivities in the order of $10^{13}-10^{17} \Omega \mathrm{m}$ [16], i.e., they are excellent electrical insulators.

\section{Rheology measurements}

Controlled shear stress (CSS) rotational rheometry was used to analyse the flow behaviour of the three base waxes and the electroconductive carbon-wax composite materials. However, the TA Instruments AR500 Advanced Rheometer that was employed has a defined upper limit to its generated shear stress; so, whilst several preliminary experiments were carried out on the A7-11 wax matrix composites, the lower working viscosity of the A7-7808 
grade made it the preferred matrix for the primary rheological studies because the use of a matrix wax with a lower working viscosity enabled results to be obtained from samples with higher electroconductive filler concentrations. A parallel plate set-up was used with a $300 \mu \mathrm{m}$ separation gap. Each electroconductive wax sample was allowed to reach a temperature of $80{ }^{\circ} \mathrm{C}$ on the Peltierheated lower plate before the upper plate was lowered to the separation distance for testing.

CSS flow ramp tests were carried out at $80 \pm 0.1{ }^{\circ} \mathrm{C}$ in order to observe the degree of thixotropic behaviour and to ascertain the viscosity of the material at a set shear stress value [17]. A two-stage stress profile was followed; a 0.779-1500 Pa upward ramp and 1500-0.779 Pa downward ramp. The experimental timeframe was set to $480 \mathrm{~s}$, with points plotted to a logarithmic preset. Half of the samples were subjected to an additional pre-shearing cycle (300 s, $750 \mathrm{~Pa}$ ) prior to flow ramp testing.

CSS temperature ramp tests were carried out to determine whether the addition of a thermally conducting electroconductive filler material would alter the temperature of the liquid to semi-liquid to solid phase transition. It is possible to deduce the phase transition temperature from the point where the sample behaviour no longer follows the Arrhenius-Frenkel-Eyring (AFE) relationship

$\eta=C e^{\left(\frac{E_{a}}{R T}\right)}$

where $\eta$ is the temperature-dependent material viscosity, $C$ is a material-specific constant, $E_{\mathrm{a}}$ is the activation energy for viscous flow, $R$ is the ideal gas constant, and $T$ is the absolute temperature. This relationship is based on flow theories considering the potential energy that must be overcome by a molecular unit to move from one equilibrium position to the next [18]. A two-stage temperature profile was followed; an initial downward temperature ramp (from 80 to $55{ }^{\circ} \mathrm{C}$ ) was followed by an upward temperature ramp (from 55 to $80{ }^{\circ} \mathrm{C}$ ), using a heating/ cooling rate of $1{ }^{\circ} \mathrm{C}$ per minute. Points were recorded in $0.5^{\circ} \mathrm{C}$ increments. CSS mode was used with constant applied shear stress values of 200, 750 and $1500 \mathrm{~Pa}$. However, only the results relating to the use of the $750 \mathrm{~Pa}$ stress parameter are presented, as the graphite-filled samples exhibited reproducible results at this stress level.

\section{Results and discussion}

Surface area, particle morphology and particle/ agglomerate size distributions of the as-received carbon powders

According to the multipoint BET analysis, the Cabot Vulcan 605 carbon black powder had a surface area of
$112 \mathrm{~m}^{2} / \mathrm{g}$ (which is in accordance with the manufacturer's information), whereas the micronised graphite had a surface area of $16 \mathrm{~m}^{2} / \mathrm{g}$.

As regards particle morphology, SEM in SEI mode showed the particle/agglomerate morphology of both carbon powders to be plate-like. Comparing the carbon black powder in Fig. 2 with the micronised graphite powder in Fig. 3, taking account of the different size scales, it can be seen that the carbon black powder exhibits a higher degree of agglomeration over a wider size range than the micronised graphite powder. In contrast, the graphite powder appears to be more loosely agglomerated over a narrower

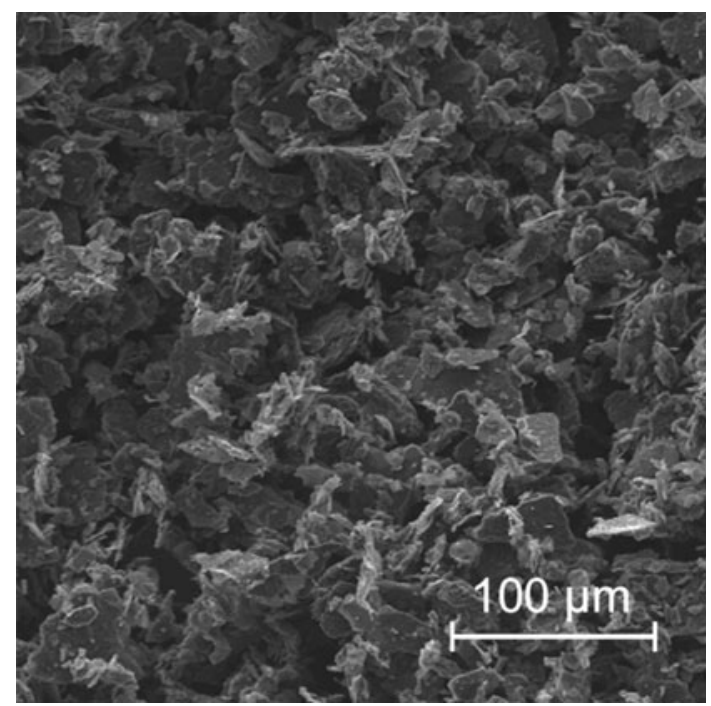

Fig. 2 SEI SEM micrograph of the as-received Vulcan XC605 carbon black (Cabot) powder

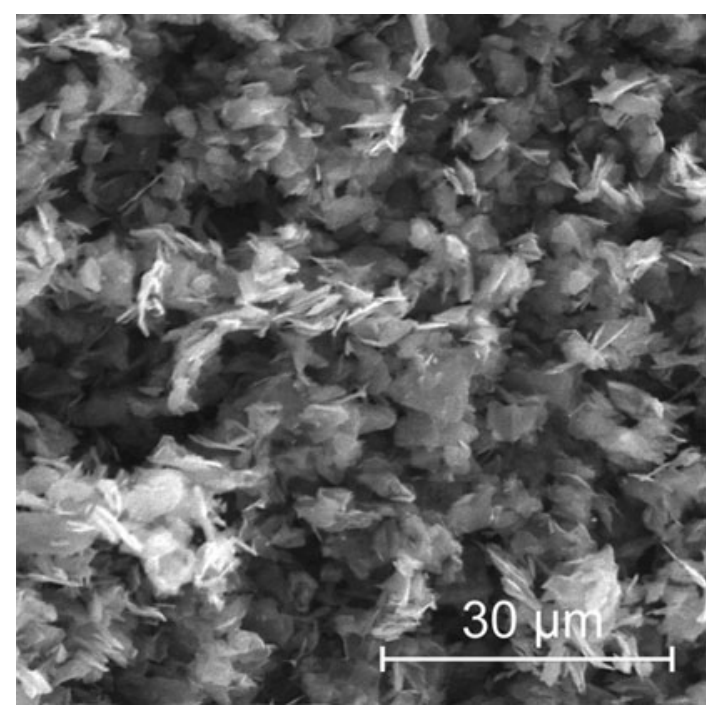

Fig. 3 SEI SEM micrograph of the as-received micronised graphite (Graphite Trading Company) powder 
size range, with the particles and agglomerates being more flaky and angular in nature.

These SEM observations are borne out by the LDPSA results, which are shown in Figs. 4, 5, 6 and 7. While it is acknowledged that (a) laser diffraction particle size distribution analysis (LDPSA) techniques cannot distinguish between dispersed particles and particle agglomerates, and (b) the particle size distribution results become subject to increasing bias and error as the particle morphology deviates further from the assumed spherical morphology, it is the comparative particle/agglomerate size distributions that are of particular interest in the present work, rather than the absolute particle sizes or agglomerate sizes.

Thus, it can be seen by comparing Figs. 4 and 5 for the micronised graphite powder suspension in ethanol that the ultrasonication cycle employed reduced the volume \% modal size from 6 to $5 \mu \mathrm{m}$ in Fig. 4 , and increased the

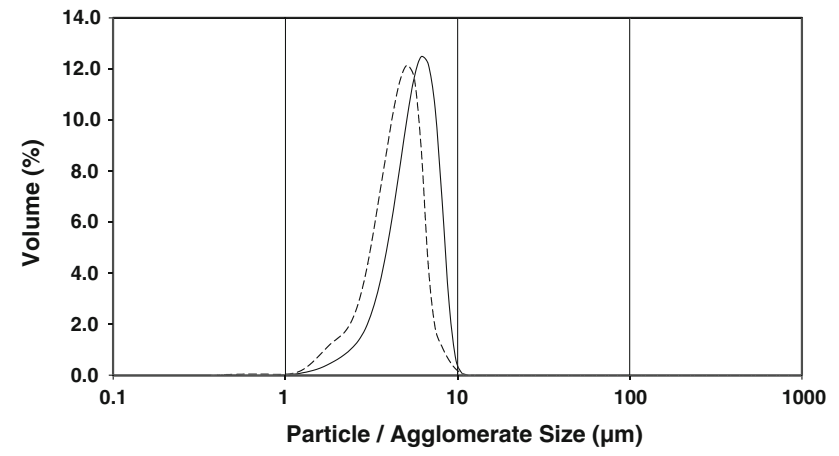

- Micronised Graphite: Unsonicated Ethanol-based Suspension

----Micronised Graphite: Sonicated Ethanol-based Suspension

Fig. 4 Laser diffraction particle size analysis of the micronised graphite powder, showing the particle/agglomerate volume $\%$ as a function of particle/agglomerate size, for sonicated and unsonicated ethanol-based suspensions

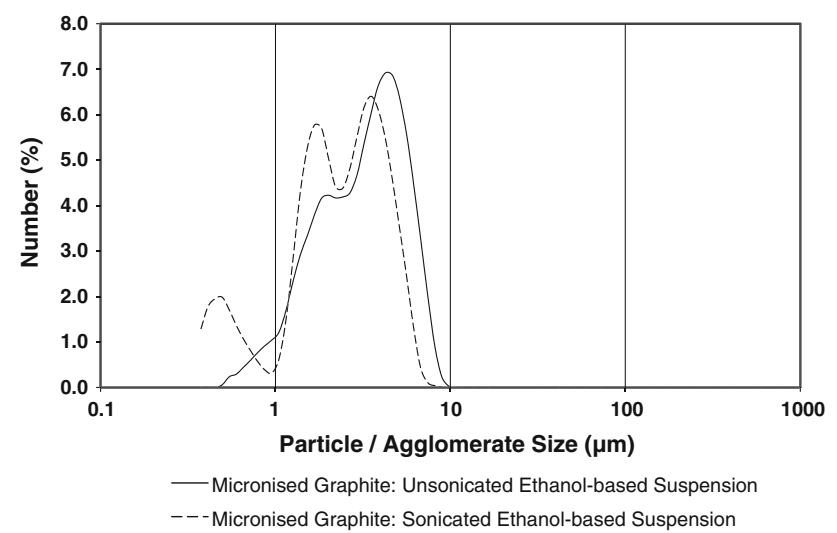

Fig. 5 Laser diffraction particle size analysis of the micronised graphite powder, showing the particle/agglomerate number $\%$ as a function of particle/agglomerate size, for sonicated and unsonicated ethanol-based suspensions

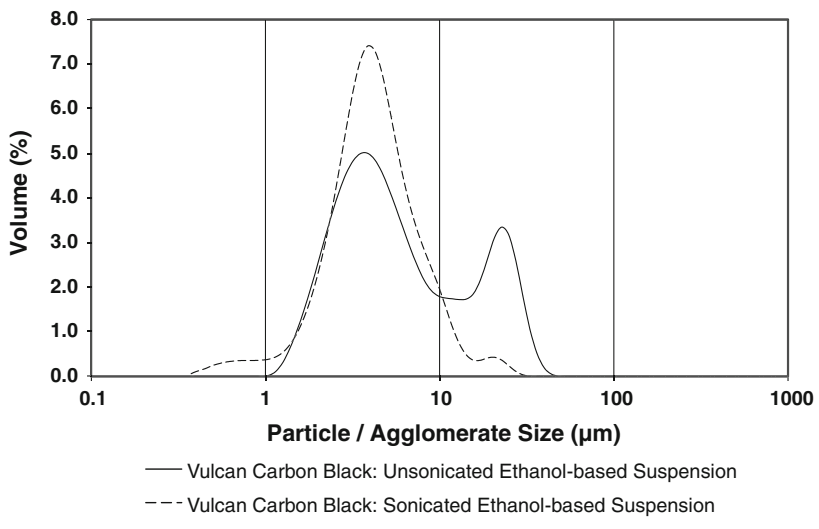

Fig. 6 Laser diffraction particle size analysis of the Vulcan carbon black powder, showing the particle/agglomerate volume $\%$ as a function of particle/agglomerate size, for sonicated and unsonicated ethanol-based suspensions

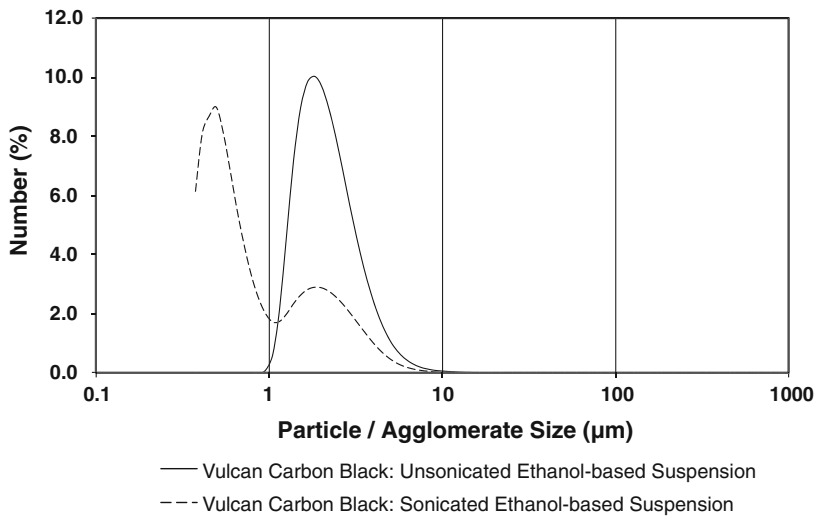

Fig. 7 Laser diffraction particle size analysis of the Vulcan carbon black powder, showing the particle/agglomerate number $\%$ as a function of particle/agglomerate size, for sonicated and unsonicated ethanol-based suspensions

number $\%$ of smaller size particle agglomerates and dispersed individual particles. The deagglomerating effect upon ultrasonication is evident in Fig. 5, where the two main peaks, with modes of 1.9 and $4.2 \mu \mathrm{m}$ shift to 1.7 and $3.3 \mu \mathrm{m}$, respectively, with a concomitant number \% decrease at $3.3 \mu \mathrm{m}$ and increase at $1.7 \mu \mathrm{m}$, and the appearance of a new number $\%$ peak at $500 \mathrm{~nm}$. These changes suggest that the ultrasonication process essentially reduced the different particle agglomerate sizes within the suspension by stripping off much smaller particle agglomerates and/or individual particles from the original particle agglomerates into the suspension. These LDPSA results confirm that the micronised graphite powder comprises individual powder particles (and particle agglomerates) smaller than $10 \mu \mathrm{m}$ in size, as stated by the manufacturer, with individual particles of $\sim 1.7-$ and $\sim 0.5-\mu \mathrm{m}$ diameter being present within the $<10-\mu \mathrm{m}$ diameter agglomerates. 
In contrast, for the carbon black powder, looking at Fig. 6, the effect of the ultrasonication cycle employed was to break up most of the particle agglomerates with a size of $22 \mu \mathrm{m}$ into agglomerates of $4 \mu \mathrm{m}$ in diameter, as well as some sub-micron size agglomerates. However, after ultrasonication, the 4- $\mu \mathrm{m}$ diameter agglomerates dominate volume \%-wise because the original $3.8 \mu \mathrm{m}$ agglomerates appear to have attracted some primary particle aggregates from the broken-up 22- $\mu \mathrm{m}$ diameter agglomerates and increased slightly in size. In contrast, Fig. 7 shows that prior to ultrasonication, the suspension was dominated number \%-wise by agglomerates between 1 and $10 \mu \mathrm{m}$ in size formed by primary particle aggregates clustering together, with the majority being $\sim 1.8 \mu \mathrm{m}$ in size.

Upon ultrasonication, the number $\%$ of primary aggregate clusters between 1 and $10 \mu \mathrm{m}$ in size is significantly reduced, although the majority that remain are still $\sim 1.8 \mu \mathrm{m}$ in size, and primary particle aggregates of $\sim 500 \mathrm{~nm}$ in size become dominant in the suspension. These LDPSA results confirm the hierarchical structure within carbon black powders, where the primary particles of carbon black invariably form submicron size very strongly bonded primary particle aggregates that (a) ordinarily cannot be broken down and (b) readily cluster together to form strongly bonded agglomerates ranging from 1 to $100 \mu \mathrm{m}$ in size, or larger.

\section{Electroconductive wax composite conductivity analysis}

As shown in Fig. 8, for both the A7-7808- and A7-TC2/Ebased micronised graphite-filled waxes, the samples with micronised graphite loadings below 14 vol\%, no resistance values could be obtained (inferring a resistance $>100 \mathrm{k} \Omega$ ), and hence no resistance data are shown, whereas for the A7-11 samples this occurred at a micronised graphite loading of less than $12 \mathrm{vol} \%$. In contrast, for the A7-7808and A7-TC2/E-based carbon black-filled waxes, no resistance values could be obtained for samples with carbon black loadings less than $15.9 \mathrm{vol} \%$, while this point was achieved for the A7-11 samples at carbon black loadings of less than $11.7 \mathrm{vol} \%$. However, it is interesting to note that, irrespective of the wax matrix, the use of carbon black as a electroconductive filler led to consistently lower resistivities at a given volume $\%$, or weight $\%$ (wt $\%$ ) above 15.9 vol\%. Furthermore, using the A7-11 wax grade as the matrix (electrically insulating) led to consistently lower resistivity values for a given percentage of electroconductive filler, micronised graphite, or carbon black.

Chan et al. [5] observed that the resistivity of carbon black-UHMW composite materials increased with temperature, and attributed this to a higher degree of intermixing of the carbon black and polymer particles in the interfacial regions as the polymer viscosity decreased with

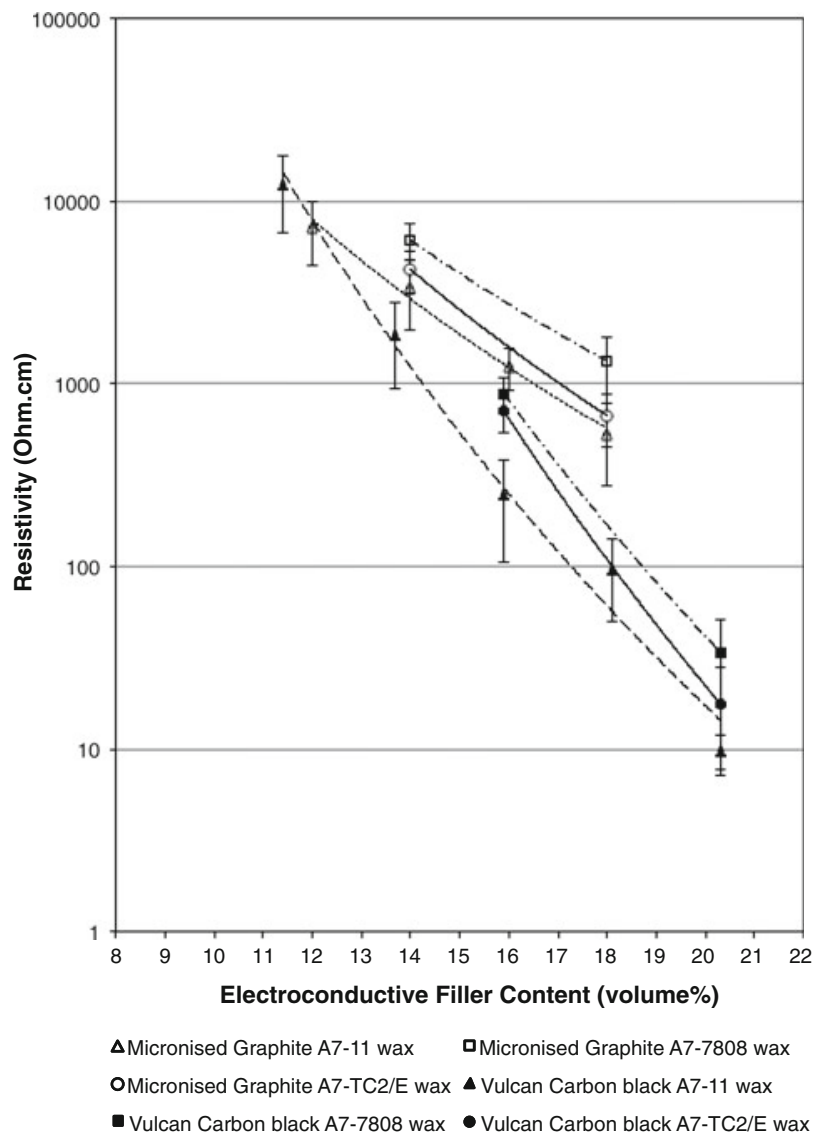

Fig. 8 Electroconductive particle-wax matrix composite sample electrical resistivity $(\Omega \mathrm{cm})$ as a function of electroconductive filler content for three wax grades, according to four-point probe tests carried out on pre-cast flat plates of electroconductive particle-filled wax

increasing temperature. Thus, in the present work, the differing viscosity-temperature dependencies of the three wax grades influence the degree of carbon black-wax intermixing achieved at the electroconductive wax formation temperature of $80^{\circ} \mathrm{C}$. The higher the degree of intermixing, the greater the dispersal, and hence isolation, of the carbon black particles within the wax matrix. This limits the formation of electroconductive pathways within the wax matrix under shear during mixing, which would otherwise reduce the resistivity of the composite material. Considering the respective base wax viscosities under a shear stress of $1500 \mathrm{~Pa}$ at $80{ }^{\circ} \mathrm{C}$, which are $0.9,0.45$ and $0.15 \mathrm{~Pa}$ s for A7-11, A7-TC2/E and A7-7808, respectively, it can be seen that the degree of intermixing increases in the order of $\mathrm{A} 7-11, \mathrm{~A} 7-\mathrm{TC} 2 / \mathrm{E}$ and $\mathrm{A} 7-7808$, i.e., of decreasing viscosity; this explains why using the A7-11 wax grade as the matrix produced the most electroconductive wax composite materials for a given amount of conductive filler, irrespective of the carbon filler material. These findings are in agreement with the work of Pinto et al. [4], who found that increasing the processing 
temperature by $15{ }^{\circ} \mathrm{C}$ (from 208 to $223{ }^{\circ} \mathrm{C}$ ) in a Nylon-6 carbon black system almost doubled the resistivity of the resulting $32 \mathrm{wt} \%$ carbon black composite. They too attributed this behaviour to reduced viscosity resulting in greater intermixing between the carbon black and polymer matrix particles in the interfacial regions, accompanied by the break-up of carbon black particle clusters, during blending.

Electroconductive wax composite rheology behaviour

\section{CSS flow ramp behaviour}

For the A7-7808 wax, shear-thinning behaviour was observed to a small extent in the wax samples over the shear stress range of the experiment $(0.1-1500 \mathrm{~Pa})$, while the emulsified A7-TC2/E wax exhibited a stronger shearthinning behaviour as the shear rate increased; however, it is the effect of the suspended electronductive filler particles/agglomerates on the material viscosity across the shear stress range that is of particular interest. The effect of suspended filler particles on the material viscosity across the shear stress range can be separated from the behaviour of the base wax itself by normalising the viscosity value of the filled wax, $\eta_{\mathrm{f}}$ with respect to the unfilled wax viscosity value, $\eta_{0}$, at each shear stress value. This gives the relative viscosity $\eta_{\mathrm{r}}$, which is defined as $\eta_{\mathrm{r}}=\eta_{\mathrm{f}} / \eta_{0}$. Furthermore, the relative viscosity can be plotted as a function of applied shear stress rather than resultant shear rate to better observe the changes in viscosity with electroconductive filler content, as well as shear-thinning behaviour over the shear stress range of the experiment $(0.1-1500 \mathrm{~Pa})$. This is shown for the micronised graphite filler in the A7-7808 wax and A7-TC2/E wax in Figs. 9 and 10, respectively, versus the carbon black filler in the A7-7808 wax and A7TC2/E wax in Figs. 11 and 12, respectively.

The presence of micronised graphite filler in both the A7-7808 and A7-TC2/E waxes increases the shear thinning nature of the wax composite material. The presence of carbon black-filler within both A7-7808 and A7-TC2/E waxes has a more marked effect than graphite on the wax composite flow behaviour. This finding that agrees with the observations of King et al. [19], who, in their study on the electrical conductivity of graphite and carbon black in polypropylene-based resins, noted that using carbon black as a filler resulted in a large increase in composite melt viscosity.

The characteristic viscosity versus shear rate curve for a shear thinning material exhibits two extremes referred to as the lower and upper Newtonian regions, i.e., the very low to low and high to very high shear rate regions, respectively [20]. The constant viscosity at very low shear rates is called the 'zero-shear viscosity' and the lower constant

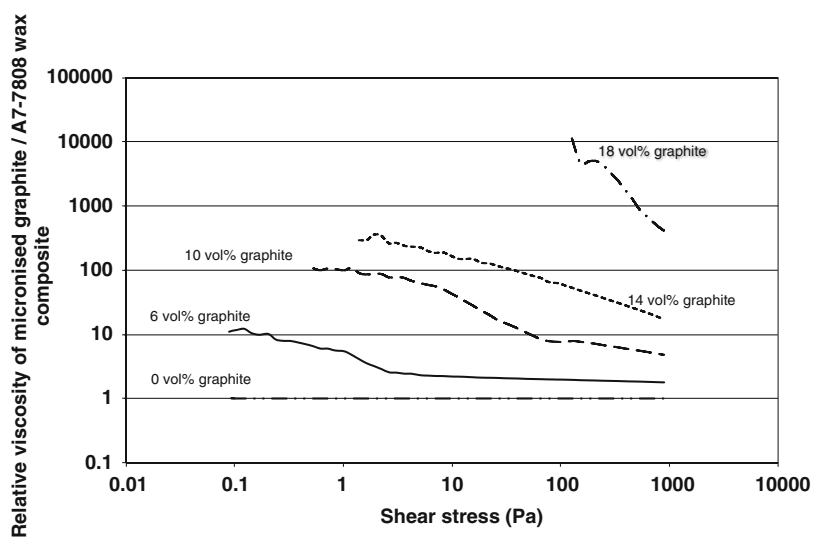

Fig. 9 Relative viscosity of the micronised graphite/A7-7808 wax composite as a function of shear stress from CSS flow ramp testing, for a range of micronised graphite concentrations (vol\%)

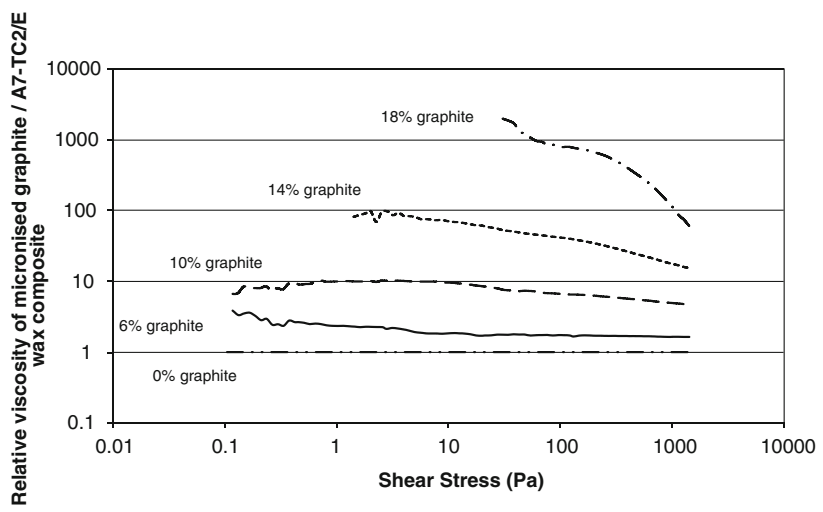

Fig. 10 Relative viscosity of the micronised graphite/A7-TC2/E wax composite as a function of shear stress from CSS flow ramp testing, for a range of micronised graphite concentrations (vol\%)

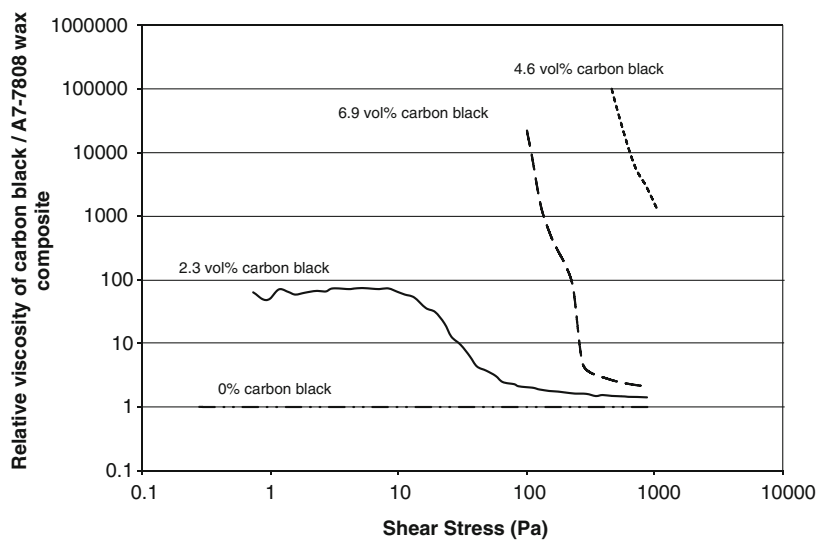

Fig. 11 Relative viscosity of the carbon black/A7-7808 wax composite as a function of shear stress from CSS flow ramp testing, for a range of carbon black concentrations (vol\%)

viscosity at very high shear rates is called the infiniteshear' viscosity. The difference between the zero-shear viscosity and infinite shear viscosity values is an indicator 


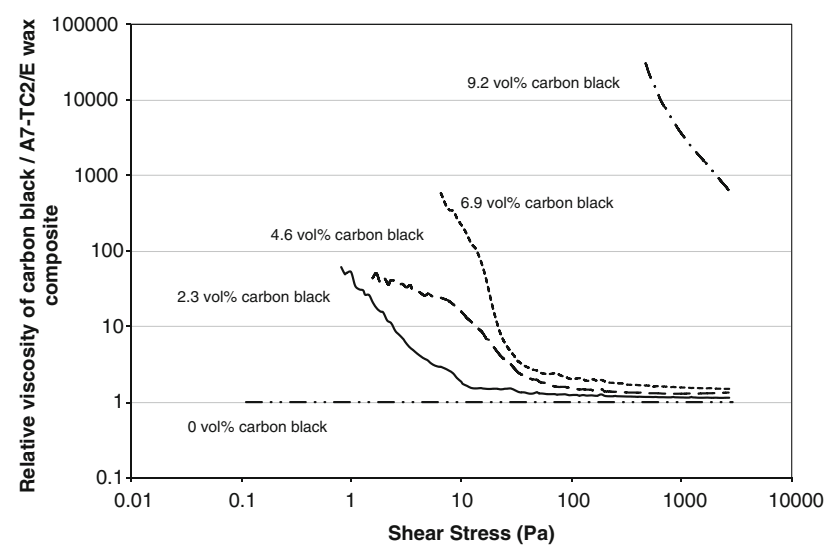

Fig. 12 Relative viscosity of the carbon black/A7-TC2/E wax composite as a function of shear stress from CSS flow ramp testing, for a range of carbon black concentrations (vol\%)

of the degree of shear-thinning behaviour. However, within the experimental range of these flow tests in the present work, no clear lower or upper Newtonian regions could be defined for any wax composition. Instead, Table 3 gives two viscosity values for each composition; the low-shear viscosity of the sample corresponds to either the viscosity at the lowest measured shear rate or at the height of the transient viscosity peak (with an associated shear rate value A), while the high-shear viscosity corresponds to the viscosity at the highest applied shear stress value in the cycle $(1500 \mathrm{~Pa})$, with an associated shear rate value $\mathrm{B}$.

As shown in Table 3, A7-7808 wax composite samples with micronised graphite filler contents greater than 6 vol\% possessed shear thinning behaviour with a yield point, whose value increases as the filler content is increased, contrasting with a yield point only becoming evident for A7-TC2/E wax composite samples with micronised graphite filler contents greater than $14 \mathrm{vol} \%$. Although the A7-7808 base wax has a lower viscosity at $80{ }^{\circ} \mathrm{C}$ than the A7-TC2/E base wax, when both the position of the yield points and the high-shear viscosity values are taken into consideration, the addition of graphite has a less marked effect on the A7-TC2/E wax. The carbon blackfilled A7-7808 wax composite samples exhibited strong shear-thinning character, with a yield point, whose values are given in Table 3. Similar non-Newtonian behaviour was observed for carbon black-filled A7-TC2/E wax composite samples. Once again, although the A7-7808 base wax has a lower viscosity at $80{ }^{\circ} \mathrm{C}$ than the A7-TC2/E base wax, the addition of carbon black has a smaller effect on the rheology of A7-TC2/E when the filler concentration was low. However, when the filler concentration was greater than $4.6 \mathrm{vol} \%$, the effect of carbon black on flow behaviour at high-shear rates was more prominent when added to A7-TC2/E.
Not all the filled samples exhibited viscosity values when lower shear stresses were used due to the presence of limiting yield points. A shear stress parameter of $750 \mathrm{~Pa}$ was therefore chosen to analyse trends, as viscosity measurements were obtained under these CSS conditions for most filled wax compositions. The aged samples underwent a pre-shear cycle for $5 \mathrm{~min}$ at $750 \mathrm{~Pa}$ prior to flow ramp testing, and displayed a consistently lower viscosity when compared to the non-aged counterparts. The viscosity disparity generally grew as the filler content was increased. The viscosity results at $750 \mathrm{~Pa}$ were compared against:

(a) The Einstein hard sphere model equation for the viscosity of a dilute suspension of non-interacting hard spheres [18]

$\eta_{\mathrm{f}}=\eta_{0}\left(1+\frac{5}{2} \varphi\right)$

where $\eta_{\mathrm{f}}$ is the viscosity of the filled wax, $\eta_{0}$ is the unfilled, i.e., base wax viscosity, and $\varphi$ is the volume fraction of filler. The Einstein equation for hard spheres only applies to low-filler concentrations $(\varphi<0.1)$, and this is the case due to the effect of particle interaction at higher concentrations.

(b) The Krieger-Dougherty [18] equation, based upon a more complex model that takes into account both particle geometry and particle interactions

$\eta_{\mathrm{f}}=\eta_{0}\left(1-\frac{\varphi}{\varphi_{\mathrm{m}}}\right)^{-[\eta] \varphi_{\mathrm{m}}}$

where $\eta_{\mathrm{f}}$ is the viscosity of the filled wax, $\eta_{0}$ is the unfilled, i.e., base wax viscosity, and $\varphi$ is the volume fraction of filler, $\varphi_{\mathrm{m}}$ is the maximum packing fraction, and $[\eta]$ is the intrinsic viscosity; $[\eta]$ describes the influence that suspended particles have on the flow behaviour of a system. For a sphere model, the $[\eta] \varphi_{\mathrm{m}}$ term [18] is $\sim 2.0$. For suspended spheres, the maximum packing fraction, $\varphi_{\mathrm{m}}$, lies between 0.52 and 0.74 .

(c) Clarke's rheology study [21] on suspensions containing glass discs with an axial ratio of 4 .

Both the Einstein hard sphere model curve and the experimental relationship between filler content and viscosity for Clarke's glass discs are shown in Figs. 13, 14 and 15 for comparative purposes. When the experimental results of the present work and those of Clarke [21] are compared, the viscosity-altering effects associated with the addition of graphite particles and glass plate particles to a matrix material diverge when the filler content is greater than $10 \mathrm{vol} \%$, as shown in Figs. 13 and 14. Above this filler content, the particle size and size distribution are known to play a greater role in the material's viscosity, and the use of smaller particles leads to higher relative 
Table 3 Selected results from controlled shear stress (CSS) flow tests on micronised graphite-filled and carbon black-filled waxes

\begin{tabular}{|c|c|c|c|c|c|c|}
\hline $\begin{array}{l}\text { Weight \% } \\
\text { filler }\end{array}$ & $\begin{array}{l}\text { Volume \% } \\
\text { filler }\end{array}$ & $\begin{array}{l}\text { Yield point } \\
(\mathrm{Pa})\end{array}$ & $\begin{array}{l}\text { Low shear viscosity } \\
\text { (Pa.s) }\end{array}$ & $\begin{array}{l}\text { Shear rate A } \\
\left(\mathrm{s}^{-1}\right)\end{array}$ & $\begin{array}{l}\text { Highest shear viscosity } \\
\text { (Pa.s) }\end{array}$ & $\begin{array}{l}\text { Shear rate B } \\
\left(\mathrm{s}^{-1}\right)\end{array}$ \\
\hline \multicolumn{7}{|c|}{ Micronised graphite-filled A7-7808 wax } \\
\hline 0 & 0 & $\mathrm{n} / \mathrm{a}$ & 0.220 & 2.025 & 0.150 & 9430 \\
\hline 12.36 & 6.0 & $\mathrm{n} / \mathrm{a}$ & 2.15 & 0.057 & 0.256 & 5590 \\
\hline 19.71 & 10.0 & 0.45 & 22.6 & 0.023 & 0.643 & 2227 \\
\hline 26.45 & 14.0 & 1.15 & 65.6 & 0.029 & 1.840 & 773.6 \\
\hline 32.65 & 18.0 & 105 & 1840 & 0.068 & 36.6 & 39.4 \\
\hline \multicolumn{7}{|c|}{ Micronised graphite-filled A7-TC2/E wax } \\
\hline 0 & 0 & $\mathrm{n} / \mathrm{a}$ & 1.80 & 0.057 & 0.456 & 3106 \\
\hline 12.36 & 6.0 & $\mathrm{n} / \mathrm{a}$ & 5.843 & 0.018 & 0.743 & 1910 \\
\hline 19.71 & 10.0 & $\mathrm{n} / \mathrm{a}$ & 11.71 & 0.052 & 2.12 & 687.0 \\
\hline 26.45 & 14.0 & 1.30 & 78.49 & 0.026 & 6.92 & 209.7 \\
\hline 32.65 & 18.0 & 27.6 & 1167 & 0.026 & 26.52 & 54.3 \\
\hline \multicolumn{7}{|c|}{ Carbon black-filled A7-7808 wax } \\
\hline 0.00 & 0.0 & $\mathrm{n} / \mathrm{a}$ & 0.273 & 0.430 & 0.1301 & 9293 \\
\hline 4.31 & 2.31 & 0.19 & 13.3 & 0.031 & 0.176 & 7490 \\
\hline 8.43 & 4.61 & 97 & 3690 & 0.027 & 0.290 & 1580 \\
\hline 12.36 & 6.90 & 390 & 16010 & 0.029 & 105.1 & 14.1 \\
\hline 16.12 & 9.17 & 930 & 46500 & 0.022 & 4940 & 0.299 \\
\hline \multicolumn{7}{|c|}{ Carbon black-filled A7-TC2/E wax } \\
\hline 0.00 & 0.0 & $\mathrm{n} / \mathrm{a}$ & 1.497 & 0.078 & 0.453 & 3224 \\
\hline 4.31 & 2.31 & 0.53 & 32.93 & 0.024 & 0.561 & 2800 \\
\hline 8.43 & 4.61 & 5.70 & 253.7 & 0.025 & 0.750 & 2110 \\
\hline 12.36 & 6.90 & 420 & 16320 & 0.029 & 761.0 & 2.10 \\
\hline 16.12 & 9.17 & 1100 & 34100 & 0.040 & 10200 & 0.157 \\
\hline
\end{tabular}

${ }^{a}$ Low-shear viscosity relates to the maximum measured viscosity in the up-curve, either at the lowest measured shear rate or at the transient viscosity peak (with an associated shear rate value A), if present

${ }^{\mathrm{b}}$ Highest shear viscosity corresponds to the sample viscosity at an applied shear stress of $\sim 1500 \mathrm{~Pa}$ (with an associated shear rate value B)

viscosities [22]. For the carbon black-filled waxes, the relative viscosity values deviated from Clarke's glass plate model when the filler content was greater than 4.6 vol\%, as shown in Fig. 15. The effect of filler agglomeration on flow behaviour within carbon black systems is believed to account for the lower divergent value, as particle agglomeration immobilises the matrix material. Using the Krieger-Dougherty equation to generate a curve that follows the experimental results can produce values for the maximum packing fraction $\left(\varphi_{\mathrm{m}}\right)$ and the intrinsic viscosity $[\eta]$, and these values could then be used to explain differences in behaviour. Sigmaplot 11.0 was used to produce values for $\varphi_{\mathrm{m}}$ and $[\eta]$ within Eq. 6 to fit the curve produced by experimental results. For iterative purposes, their initial values were set to those for a glass plate system $\left(\varphi_{\mathrm{m}}=0.382 ;[\eta]=9.87\right)[18,21]$. Table 4 displays the results of the Krieger-Dougherty curve fitting.

Figure 13 shows the relationship between viscosity and micronised graphite-filler content at $80{ }^{\circ} \mathrm{C}$ for both aged and non-aged graphite-filled A7-7808 waxes, along with the fitted Krieger-Dougherty curves. The corresponding value of each variable in Eq. 6 is shown in Table 4, and gives values for $\varphi_{\mathrm{m}}$ which are only slightly above the

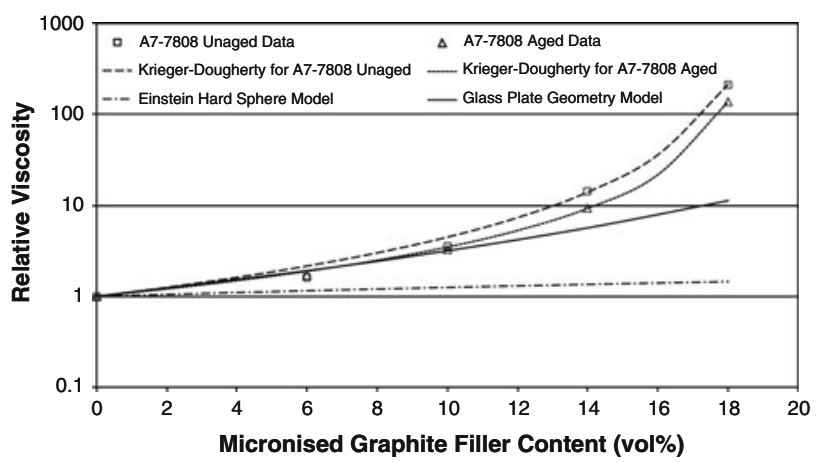

Fig. 13 Relative viscosity as a function of micronised graphite filler content for both aged and non-aged graphite-filled A7-7808 waxes $\left(750 \mathrm{~Pa}, 80^{\circ} \mathrm{C}\right)$. A Krieger-Dougherty curve for each data set is shown. Einstein's hard sphere model curve and an experimental curve for glass plates (after Clarke [21]) are also shown 


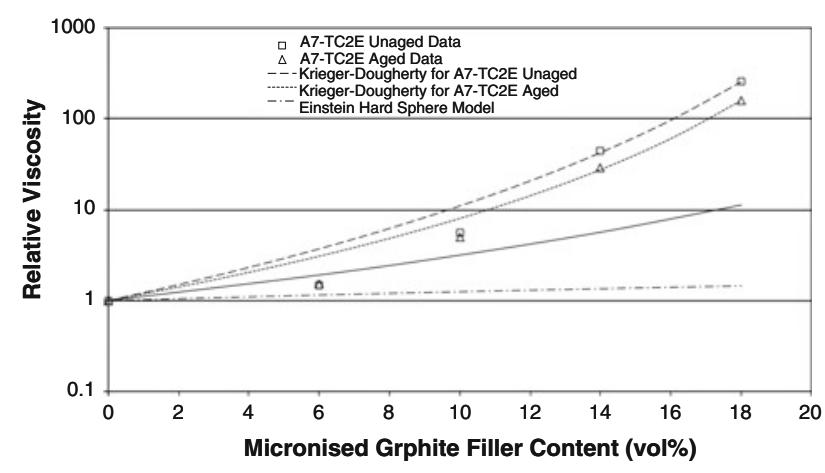

Fig. 14 Relative viscosity as a function of micronised graphite filler content for both aged and non-aged graphite-filled A7-TC2/E waxes $\left(750 \mathrm{~Pa}, 80^{\circ} \mathrm{C}\right)$. A Krieger-Dougherty curve for each data set is shown, as well as Einstein's hard sphere model curve and an experimental curve for Clarke's glass plates [21]

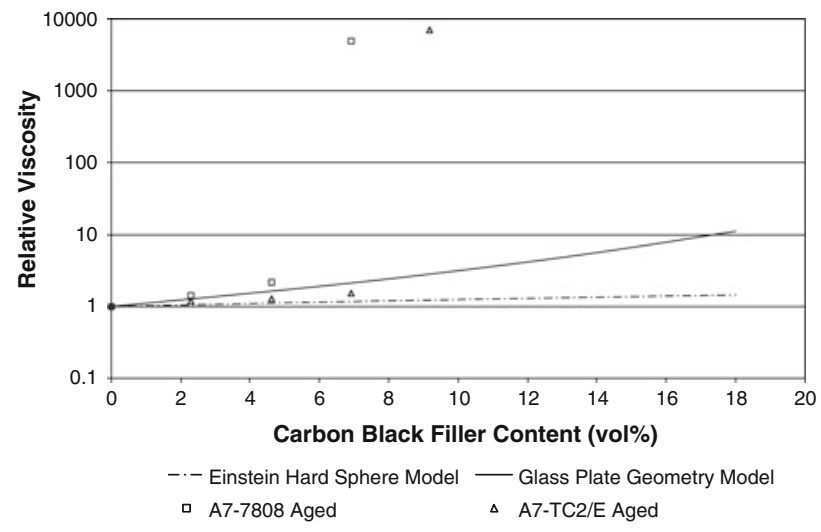

Fig. 15 Relative viscosity as a function of carbon black filler content for both aged carbon black-filled A7-7808 and A7-TC2/E waxes $\left(750 \mathrm{~Pa}, 80^{\circ} \mathrm{C}\right)$, versus the Einstein hard sphere model and an experimental curve for glass plates (after Clarke [21])

experimental range $\left(\varphi_{\mathrm{m}}=0.1948\right.$ with no pre-shear; $\varphi_{\mathrm{m}}=0.1899$ with pre-shear). The preliminary experiments associated with graphite-filled wax composite fabrication used filler additions higher than those used here; however, the graphite particle distribution homogeneity could not be maintained. The associated high goodness-offit parameter $R^{2}$ values show that this filled wax system closely follows the Krieger-Dougherty relationship.

Figure 14 shows the same relationship for both aged and non-aged micronised graphite-filled A7-TC2/E waxes. Altering the Krieger-Dougherty parameters $[\eta]$ and $\varphi_{\mathrm{m}}$ did not, however, lead to a theoretical curve shape that fitted the experimentally derived plotted points for both low- and high-filler contents. Relative viscosity values at 6 and 10 vol\% were lower than model behaviour predictions, explaining the lower $R^{2}$ values of 0.9992 and 0.9993 , respectively, at $80{ }^{\circ} \mathrm{C}$. Higher $\varphi / \varphi_{\mathrm{m}}$ values increase the relative viscosity of the filled material [18]. It is important to note that both graphite and carbon black powders are plate-like in nature, and such shapes trap the wax on their surfaces, which contributes to a reduction in $\varphi_{\mathrm{m}}$.

Figures 16 and 17 show graphically the effect of temperature on flow curve-derived relative viscosity at $750 \mathrm{~Pa}$ when A7-7808 and A7-TC2/E waxes were used, respectively. The Krieger-Dougherty fitting should be independent of temperature, and when the A7-7808 grade wax was used as the base wax, the effect of graphite addition on relative viscosity changed little over the $15{ }^{\circ} \mathrm{C}$ temperature range studied. From the Sigmaplot data in Table 4, $[\eta]$ increased with temperature, forming a plateau at $75{ }^{\circ} \mathrm{C}$ and above. The influence of graphite filler on the system rheology may therefore vary over the melting range. Values for the maximum packing fraction, $\varphi_{\mathrm{m}}$, also increase with temperature, and go from $17 \mathrm{vol} \%$ at $65{ }^{\circ} \mathrm{C}$ to $19.5 \mathrm{vol} \%$ at $80{ }^{\circ} \mathrm{C}$. The relative viscosity increases with temperature, when micronised graphite was added to A7-TC2/E at higher filler concentrations, as shown in Fig. 17. Again from Table $4,[\eta]$ generally increases with temperature, although $\varphi_{m}$ values show no clear temperature dependence. For carbon-black filled waxes, a temperature dependent relative viscosity at $750 \mathrm{~Pa}$ could not be produced due to the presence of limiting yield points when the temperature was below $80^{\circ} \mathrm{C}$.

To explain the values given in Table 4 , the contributing factors that affect the variables $[\eta]$ and $\varphi_{\mathrm{m}}$ must be considered. Particle shape plays an important role in the rheology of suspensions, and influences the maximum packing fraction $\left(\varphi_{\mathrm{m}}\right)$ and intrinsic viscosity $[\eta]$ parameters of Eq. 6. The value of $[\eta]$ is based on the filler's contribution to the viscosity of the solution, and according to Einstein's model, has a value of 2.5 for solid spheres. Its value is very sensitive to the axial ratio of spheroids, and for nonspherical geometries, specific empirical relationships have been ascertained by Barnes [23] for discs or plates and for rods

$$
\begin{array}{lll}
\text { discs or plates : } & & {[\eta]=\left(\frac{3}{10}\right) \cdot(\text { axial ratio })} \\
\text { rods: } & {[\eta]=\left(\frac{7}{100}\right) \cdot(\text { axial ratio })^{\frac{5}{3}}}
\end{array}
$$

For both systems, the intrinsic viscosity can be determined by measuring the reduced viscosity, $\eta_{\text {red }}$ given by $\left(\eta_{\mathrm{r}}-1\right) / c$, where $c$ is the mass concentration of filler in the suspension, at a number of different concentrations. By extrapolating the reduced viscosity relationship to zero concentration, a value for $[\eta]$ is obtained. According to Mezger [17], seven or more concentrations need to be tested to use this method; unfortunately, time constraints excluded its use in the present work. The axial ratio of the plate-like graphite particles could be estimated from SEM micrographs, e.g., 
Table 4 Values for the intrinsic viscosity [ $\eta]$, maximum packing fraction $\varphi_{\mathrm{m}}$ and goodness-of-fit parameter $R^{2}$, obtained by fitting the KriegerDougherty relationship against data obtained from flow curves in a rotational rheometer at varying temperatures and pre-shear states

\begin{tabular}{|c|c|c|c|c|c|c|}
\hline Base wax & Temperature $\left({ }^{\circ} \mathrm{C}\right)$ & Ageing/pre-shear & {$[\eta]$} & $\varphi_{\mathrm{m}}$ & $\varphi_{\mathrm{m}}[\eta]$ & $R^{2}$ \\
\hline \multicolumn{7}{|c|}{ Micronised graphite } \\
\hline A7-7808 & 65 & No & 8.98594 & 0.1707 & 1.5339 & 0.9989 \\
\hline A7-7808 & 65 & $750 \mathrm{~Pa}, 300 \mathrm{~s}$ & 7.32421 & 0.1681 & 1.2312 & 0.9987 \\
\hline A7-7808 & 70 & No & 9.66890 & 0.1791 & 1.7319 & 0.9992 \\
\hline A7-7808 & 70 & $750 \mathrm{~Pa}, 300 \mathrm{~s}$ & 7.96751 & 0.1710 & 1.3624 & 0.9990 \\
\hline A7-7808 & 75 & No & 10.7291 & 0.1938 & 2.0793 & 1.0000 \\
\hline A7-7808 & 75 & $750 \mathrm{~Pa}, 300 \mathrm{~s}$ & 9.15684 & 0.1900 & 1.7398 & 0.9978 \\
\hline A7-7808 & 80 & No & 10.6776 & 0.1948 & 2.080 & 1.0000 \\
\hline A7-7808 & 80 & $750 \mathrm{~Pa}, 300 \mathrm{~s}$ & 8.77303 & 0.1899 & 1.666 & 1.0000 \\
\hline \multicolumn{7}{|c|}{ Micronised graphite } \\
\hline A7-TC2/E & 70 & No & 16.89654 & 0.3499 & 5.9121 & 0.9958 \\
\hline A7-TC2/E & 70 & $750 \mathrm{~Pa}, 300 \mathrm{~s}$ & - & - & - & - \\
\hline A7-TC2/E & 75 & No & 15.30119 & 0.2520 & 3.8559 & 0.9991 \\
\hline A7-TC2/E & 75 & $750 \mathrm{~Pa}, 300 \mathrm{~s}$ & 13.2488 & 0.2038 & 2.7001 & 0.9999 \\
\hline A7-TC2/E & 80 & No & 19.4670 & 0.2852 & 5.5521 & 0.9992 \\
\hline A7-TC2/E & 80 & $750 \mathrm{~Pa}, 300 \mathrm{~s}$ & 16.4693 & 0.2589 & 4.2639 & 0.9993 \\
\hline \multicolumn{7}{|c|}{ Vulcan carbon black } \\
\hline A7-7808 & 80 & $750 \mathrm{~Pa}, 300 \mathrm{~s}$ & 63.4966 & $0.089^{\mathrm{a}}$ & 5.6512 & 0.9998 \\
\hline \multicolumn{7}{|c|}{ Vulcan carbon black } \\
\hline A7-TC2/E & 80 & $750 \mathrm{~Pa}, 300 \mathrm{~s}$ & 65.1056 & $0.089^{\mathrm{a}}$ & 5.7944 & 0.9998 \\
\hline
\end{tabular}

All measurements were taken at a shear stress of $750 \mathrm{~Pa}$

${ }^{a}$ In these results, the plots would not converge on a value for $\varphi_{\mathrm{m}}$, and so the maximum value tested in that respective study was used as a minimal value

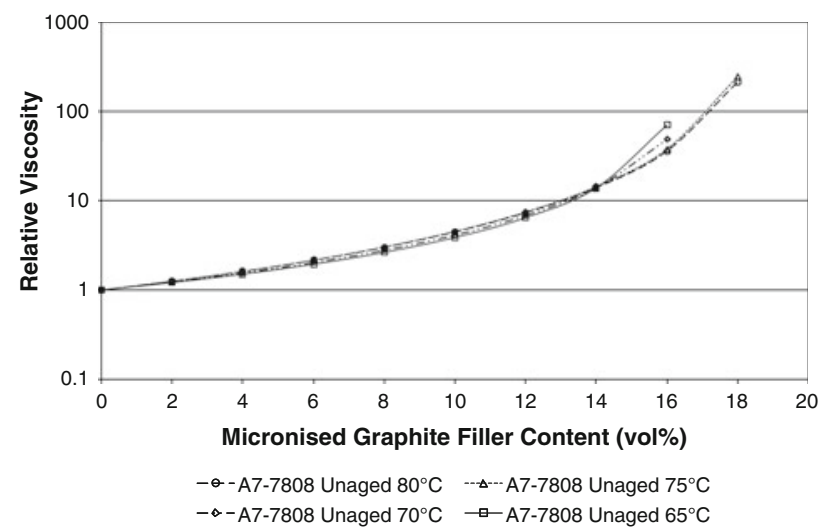

Fig. 16 Relative viscosity, determined using the Krieger-Dougherty model, as a function of micronised graphite filler content for unaged graphite-filled A7-7808 waxes at differing temperatures, under a shear stress of $750 \mathrm{~Pa}$

as shown in Fig. 3, with the estimated axial ratios used to explain any changes. The particles appear to have a fine flake-like structure, with estimated axial ratios between 4 and 10. According to Eq. 7, an axial ratio of 4 should lead to a $[\eta]$ value of 1.2 , whilst an axial ratio of 10 should lead to a $[\eta]$ value of 3 . The glass plates in suspension studied by Clarke [21] were stated as having an axial ratio of 4 , and

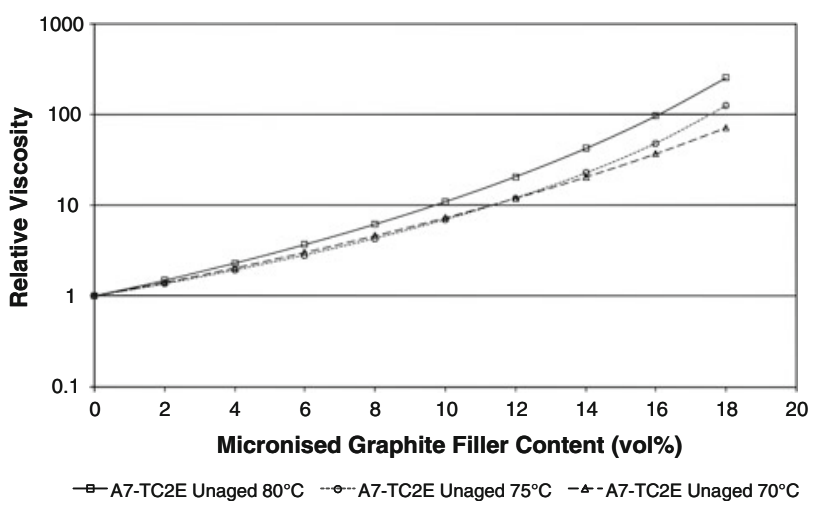

Fig. 17 Relative viscosity, determined using the Krieger-Dougherty model, as a function of micronised graphite filler content for unaged graphite-filled A7-TC2/E waxes at differing temperatures, under a shear stress of $750 \mathrm{~Pa}$

yet a $[\eta]$ value of 9.87 was given as the Krieger-Dougherty parameter. Within the present work, similar $[\eta]$ values to those obtained by Clarke were obtained when the A7-7808 wax was used to suspend graphite, as shown in Table 4. However, values for $[\eta]$ were roughly double this figure, when the water-emulsified A7-TC2/E wax was used to suspend the same micronised graphite powder. The axial ratio of the graphite particles themselves should not change 
in these two environments, although the base wax may have an effect on filler morphology. Interactions between filler and water in the A7-TC2/E wax may play an important role, along with wettability differences and agglomeration level. The maximum packing fraction $\left(\varphi_{\mathrm{m}}\right)$ represents the highest volume of particles that can be added to a suspending liquid medium. It is possible to think of this limit in terms of the free space around particles. At $\varphi_{\mathrm{m}}$, there is no space for them to move, to shift past one another, and thus generate flow. The particle shape, agglomeration level, size and size distribution all have an effect on this value. The factors listed above can be used to explain the difference between the $\varphi_{m}$ values obtained in the present work and those given for the glass plate system studied by Clarke [21]. A far higher $\varphi_{\mathrm{m}}$ value of 0.382 was obtained for the glass plate system, compared with $\sim 0.195$ for graphite-filled A7-7808 wax and $\sim 0.28$ for graphitefilled A7-TC2/E wax at $80{ }^{\circ} \mathrm{C}$. When a powder with a finer particle size is used for a given filler volume, there would naturally be a higher number of particles present, which results in a greater number of particle-particle interactions and an increased resistance to flow. According to Pal et al. [22], this effect becomes less marked when samples are tested at high shear, which suggests that particle-particle interactions are relatively weak and can be broken down at high-shear rates. The LDPSA results in the "Surface area, particle morphology and particle/agglomerate size distributions of the as-received carbon powders" section confirm that the micronised graphite powder dispersed in ethanol comprises individual powder particles (and particle agglomerates) smaller than $10 \mu \mathrm{m}$ in size, as stated by the manufacturer. With individual particles of $\sim 1.7$ - and $\sim 0.5-\mu$ m diameter being present within the less than $10-\mu \mathrm{m}$ diameter agglomerates; the size range is far removed from the 100 by $400 \mu \mathrm{m}$ glass plates employed by Clarke [21]. The cluster size when the graphite or carbon black is dispersed within the wax itself is at present unknown.

\section{CSS temperature ramp behaviour}

Above the melting temperature of the wax, it can be appreciated that the viscosity $(\eta)$ follows the AFE relationship, shown in Eq. 4. The temperature ramp results were fitted against an Arrhenius equation plot for each test using Sigmaplot 11.0. It was possible to arrive at a temperature range for each composition where liquid-phase AFE behaviour was observed in the decreasing temperature and increasing temperature ramps, as shown in Table 5. It can be seen from Table 5 that all samples start to obey the AFE relationship for the liquid phase at a similar temperature.

When micronised graphite was used as a filler in A77808 , the onset (heating curve) and offset (cooling curve) temperatures for AFE behaviour were unaffected by filler content. However, when micronised graphite was used as a filler in A7-TC2/E, it can be seen that although the offset (cooling curve) temperatures for AFE behaviour were not affected by filler content to within $1{ }^{\circ} \mathrm{C}$, the onset (heating curve) temperatures for AFE behaviour increased slightly with filler content by $4.5^{\circ} \mathrm{C}$ for 14 vol\% filler.

When carbon black was used as the conductive filler in the samples for the temperature ramp experiments, preshear cycles were put in place, as the effect of cluster breakdown under shear would lead to sizeable curve changes during the initial stages of each experiment. When carbon black was used as a filler in A7-7808, the onset (heating curve) and offset (cooling curve) temperatures for AFE behaviour were unaffected by filler content, with the temperature ranges being the same as that for the micronised graphite filler. However, when carbon black was used as a filler in A7-TC2/E, it can be seen that although the offset (cooling curve) temperatures for AFE behaviour were not affected by filler content to within $1{ }^{\circ} \mathrm{C}$, there is a tendency for the offset temperature to be around a degree higher than those for the micronised graphite. Furthermore, as for the micronised graphite, the onset (heating curve) temperatures for AFE behaviour increased with filler content, but by $8{ }^{\circ} \mathrm{C}$ for 6.9 vol $\%$ filler and with a tendency for the onset temperature to be around $4{ }^{\circ} \mathrm{C}$ higher than those for the micronised graphite.

The reason for there being no change with filler content and type for A7-7808 (at least within the range of data obtainable) cannot be fully determined without detailed compositional information on each wax grade, which is unknown due to commercial confidentiality. When micronised graphite and carbon black are used as filler materials within the water-emulsified A7-TC2/E wax grade, the solid-liquid transition region is shifted to higher temperatures during heating compared with during cooling. On heating, the presence of filler within the A7TC2/E appears to inhibit the flow of the base wax to a greater degree. The lower viscosity of the unfilled A77808 wax may have an influential effect, since as the temperature is increased during the heating ramp, the viscosity decreases, and so an increase in shear rate would enable better intermixing. This intermixing would reduce the temperature inhomogeneity within the sample, and so lead to a heating curve that more closely follows the cooling curve. It would therefore be the material's viscosity at the lowest point on the cooling ramp that has an effect on the heating curve. In addition, when CSS temperature ramps were carried out on fresh cycles, using a temperature ramp rate of $0.5{ }^{\circ} \mathrm{C}$ per minute instead of the $1{ }^{\circ} \mathrm{C}$ per minute previously used, no significant changes were observed. 
Table 5 Temperature range for each micronised graphite-filled and carbon black-filled A7-7808 and A7-TC2/E wax composition where Sigmaplot-derived AFE behaviour is observed in the liquid

\begin{tabular}{|c|c|c|c|c|c|}
\hline & \multicolumn{5}{|c|}{ Volume $\%$ micronised graphite filler } \\
\hline & 0.0 & 6.0 & 10.0 & 14.0 & 18.0 \\
\hline A7-7808 wax & $\left({ }^{\circ} \mathrm{C}\right)$ & $\left({ }^{\circ} \mathrm{C}\right)$ & $\left({ }^{\circ} \mathrm{C}\right)$ & $\left({ }^{\circ} \mathrm{C}\right)$ & \\
\hline Cooling curve AFE behaviour & $80-63$ & $80-63$ & $80-63$ & $80-63$ & - \\
\hline Heating curve AFE behaviour & $64-80$ & $64-80$ & $64-80$ & $64-80$ & - \\
\hline \multicolumn{6}{|l|}{ A7-TC2/E wax } \\
\hline Cooling curve AFE behaviour & $80-68$ & $80-67$ & $80-67.5$ & $80-68$ & - \\
\hline \multirow[t]{3}{*}{ Heating curve AFE behaviour } & $68.5-80$ & $70.5-80$ & $71.5-80$ & $73-80$ & - \\
\hline & \multicolumn{5}{|c|}{ Volume \% Vulcan XC605 carbon black filler } \\
\hline & 0.0 & 2.3 & 4.6 & 6.9 & 9.2 \\
\hline A7-7808 wax & $\left({ }^{\circ} \mathrm{C}\right)$ & $\left({ }^{\circ} \mathrm{C}\right)$ & $\left({ }^{\circ} \mathrm{C}\right)$ & $\left({ }^{\circ} \mathrm{C}\right)$ & \\
\hline Cooling curve AFE behaviour & $80-63$ & $80-63$ & $80-63$ & $80-63$ & - \\
\hline Heating curve AFE behaviour & $64-80$ & $64-80$ & $64-80$ & $64-80$ & - \\
\hline \multicolumn{6}{|l|}{ A7-TC2/E wax } \\
\hline Cooling curve AFE behaviour & $80-68$ & $80-68.5$ & $80-68.5$ & $80-69$ & - \\
\hline Heating curve AFE behaviour & $68.5-80$ & $74.5-80$ & $75.5-80$ & $76.5-80$ & - \\
\hline
\end{tabular}

Results from each decreasing temperature ramp and increasing temperature ramp are shown

\section{Conclusions}

1. For a nominal 16 vol\% carbon black content, the average electrical resistivity was 240, 710 and $880 \Omega \mathrm{cm}$ for A7-11, A7-TC2/E and A7-7808, respectively. For a nominal $18 \mathrm{vol} \%$ micronised graphite content, the average resistivity was 530,970 and $1300 \Omega \mathrm{cm}$ for A7-11, A7-TC2/E and A7-7808, respectively. Furthermore, in the case of A7-11, a $16 \mathrm{vol} \%$ content of micronised graphite produced an average electrical resistivity of $1200 \Omega \mathrm{cm}$.

2. While the Blayson Olefines A7-7808 wax was found to improve the dispersion characteristics of either filler (i.e., Vulcan XC605 Carbon Black [Cabot Co.] and Micronised graphite [Graphite Trading Co.]) within the carbon powder-wax composite material at any given working temperature, the A7-7808 wax composites exhibited consistently higher resistivities across the range of carbon black and micronised graphite filler concentrations. From this, it can be deduced that when the matrix wax has a low working viscosity, the particles are less likely to segregate to the carbon filler/wax matrix interfacial regions during cooling. Consequently, lower sample electrical conductivities will result, as the carbon particles are not ordered within the percolation network.

3. Rheologically, the addition of micronised graphite filler to both the Blayson Olefines A7-7808 straight and water-emulsified A7-TC2/E investment casting waxes produced carbon-wax composites that followed the Krieger-Dougherty relationship for agglomerated plate-shaped particles. This result is noteworthy because being able to understand, predict and hence control the micronised graphite-wax composite rheology is important, given that investment casting waxes are invariably injection moulded to form the investment casting wax patterns.

In summary, these conclusions regarding the electroconductivity and rheological behaviour of the said carboninvestment casting wax composites are a step towards confirming the feasibility of developing EPD as a promising technique for forming the investment casting shell mould ceramic face-coat. This is because, in comparison with the conventional slurry dip-coating process, EPD has the potential to (a) increase dimensional tolerances in the resultant casting and (b) form a uniform face-coat on the entire mould-surface of complex shell moulds, including the small and/or complex cavities that are a problem for conventional dip-coating. This also offers the possibility of eliminating the need for some intricate and/or delicate ceramic cores, and hence, the attendant problems their usage could bring.

Acknowledgements The authors would like to thank the European Investment Casters' Federation (EICF) Project Consortium and its members for providing financial and in-kind support to the research project. The EPSRC DTA Scheme (EPSRC Grant No. EP/P503922/1), the University of Birmingham, and the School of Metallurgy and Materials are gratefully acknowledged for their provision of a $\mathrm{PhD}$ studentship to P.J. Roach, as well as for the laboratory facilities. The authors also wish to thank Blayson Olefines Ltd., for their information 
on wax melting procedures, and for the waxes; and Cabot, for the Vulcan carbon black powder samples.

Open Access This article is distributed under the terms of the Creative Commons Attribution License which permits any use, distribution, and reproduction in any medium, provided the original author(s) and the source are credited.

\section{References}

1. Wolz S (2009) US Patent Application No: 20090255813

2. Uchikoshi T, Furumi S, Shirahata N, Suzuki TS, Sakka Y (2008) J Am Ceram Soc 91(5):1674

3. Wang K.-C., Hu C.-M. (2003) US Patent No: 6623662

4. Pinto G, López-González C, Jiménez-Martín A (1999) Polym Compos 20(6):804

5. Chan Chi-Ming, Cheng Chi-Leung, Yuen MMF (1997) Polym Eng Sci 37(7):1127

6. Guo-Hua Chen, Da-Jun Wu, Wen-Gui Weng, Bin He, Wen-li Yan (2001) Polym Int 50:980

7. Chakrabarty RK, Bardhan KK, Basu A (1991) Phys Rev B 44(13):6773

8. Piwonka TS, Woodbury KA, Wiest JM (2000) Mater Des 21(4):365

9. Grimmett G (1989) Percolation. Springer, New York

10. McLachlan DS, Blaszkiewicz M, Newnham RE (1990) J Am Ceram Soc 73(8):2187

11. Scarisbrick RM (1973) J Phys D Appl Phys 6:2098
12. Kusy RP (1977) J Appl Phys 48(12):5301

13. Carbon Black User's Guide, International Carbon Black Association (June 2004). http://www.carbon-black.org/user_guide. html. Accessed 2 May 2013

14. Uhlir A (1955) Bell Syst Tech J 34(1):105. http://www3. alcatel-lucent.com/bstj/vol34-1955/articles/bstj34-1-105.pdf. Accessed 2 May 2013

15. Geometric factors in four point resistivity measurement (1968) Bulletin No. 472-13, 2nd edn. Haldor Topsoe, Semiconductor division, Vedbaek, Denmark. http://www.four-point-probes.com/ haldor.html\#hal. Accessed 2 May 2013

16. Kaye GWC, Laby TH (1995) Tables of physical and chemical constants: 2.6.3 electrical insulating materials, 16th edn. http:// www.kayelaby.npl.co.uk/general_physics/2_6/2_6_3.html Accessed 2 May 2013

17. Mezger TG (2006) The rheology handbook: for users of rotational and oscillatory rheometers. Vincentz Network GmbH \& Co KG, Hannover

18. Barnes HA, Hutton JF, Walters K (1997) An introduction to rheology, vol 3. Elsevier, Amsterdam

19. King JA, Johnson BA, Via MD, Ciarkowski CJ (2009) J Appl Polym Sci 112(1):425

20. Elias HG (2003) An introduction to plastics. Wiley, New York, p 136

21. Clarke B (1967) Chem Eng Res Des 45a:251

22. Pal R, Bhattacharya SN, Rhodes E (1986) Can J Chem Eng 64(1):3

23. Barnes HA (1981) Dispersion rheology 1980: a Survey of Industrial Problems and Academic Progress, R Soc Chem, Industrial Division, Burlington House, London, p 31 